\title{
Analysis of the front structure of EAS with the HADES tRPC wall
}

\author{
Alberto Blanco ${ }^{a}$, Daniel Belver ${ }^{b}$, Pablo Cabanelas ${ }^{b}$, José Díaz ${ }^{c}$, Paulo Fonte ${ }^{a, d}$, \\ Juan A. Garzón ${ }^{b}$, Alejandro Gil ${ }^{c}$, Diego González-Díaz ${ }^{e}$, Wolfgang Koenig ${ }^{f}$, Georgy \\ Kornakov $^{* a, b}$, Burkhard Kolb ${ }^{f}$, Luís Lopes ${ }^{a}$, Marek Palka ${ }^{g}$, Americo Pereira $^{a}$, \\ Michael Traxler ${ }^{f}$, Peter Zumbruch ${ }^{f}$ \\ ${ }^{a}$ Laboratorio de Instrumentacão e Física Experimental de Partículas, LIP, Coimbra, Portugal \\ ${ }^{b}$ LabCAF, Universidade de Santiago de Compostela, USC, Santiago de Compostela, Spain \\ ${ }^{c}$ Instituto de Física Corpuscular, IFIC, Valencia, Spain \\ ${ }^{d}$ ISEC-Instituto Superior de Engenharia de Coimbra, Coimbra, Portugal \\ ${ }^{e}$ Laboratorio de Física Nuclear y Altas Energías, Universidad de Zaragoza, Zaragoza, Spain \\ ${ }^{f}$ GSI Helmholtzzentrum für Schwerionenforschung GmbH, Darmstadt, Germany \\ ${ }^{g}$ Smoluchowski Institute of Physics, Jagiellonian University of Cracow, Poland \\ E-mail: georgui.kornakoveusc.es
}

In the last months of 2009, during the commissioning of the Resistive Plate Chambers (RPC) Time Of Flight (TOF) wall for the High-Acceptance DiElectron Spectrometer (HADES) at the GSI (Darmstadt, Germany), more than 500 millions of cosmic ray events were recorded with a time resolution better than $100 \mathrm{ps}$, a reconstructed position resolution of the order of a few $\mathrm{cm}^{2}$ and with granularities ranging from $\sim 25 \mathrm{~cm}^{2}$ to $\sim 270 \mathrm{~cm}^{2}$. Events were recorded with pairs of detectors of $1.25 \mathrm{~m}^{2}$, stacked horizontally and separated by a distance of $\sim 35 \mathrm{~cm}$. We have analysed the performances that such setup offers for the study of cosmic rays and the microstructure of the cosmic ray-induced Extended Air Showers (EAS) fronts.

XI workshop on Resistive Plate Chambers and Related Detectors - RCP2012,

February 5-10, 2012

INFN Laboratori Nazionali di Frascati Italy

* Speaker. 


\section{Introduction}

The HADES (High-Acceptance DiElectron Spectrometer) spectrometer at GSI-SIS [1] was upgraded in 2009[2] in order to allow exploration of the dielectron production channel in heavy nuclei collisions. For such a purpose, an inner ToF Wall based on shielded timing RPCs [3] was designed[4], built and finally commissioned in a cosmic ray (CR) test [5].

Recently, some proposals have been presented to use RPC-based detectors as tracking and timing devices in order to measure CR showers [6][7]. Here, we have estimated the performance of the HADES RPCs as a detector with capability to analyse both time and position microstructure of cosmic ray showers. Among $\sim 600$ Mevent recorded, a selection of $\sim 40$ Mevent, corresponding to a five days continuous run under stable conditions, has been chosen for analysis. The mean trigger rate was around $100 \mathrm{~Hz} / \mathrm{m}^{2}$. A description of all the calibration and synchronization procedures, track candidate finder and some analysis results are presented.

\section{Setup description, calibration and synchronization}

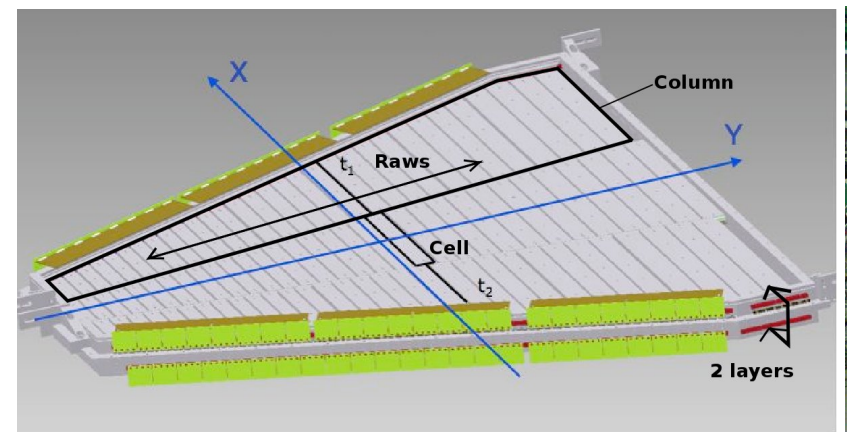

(a)

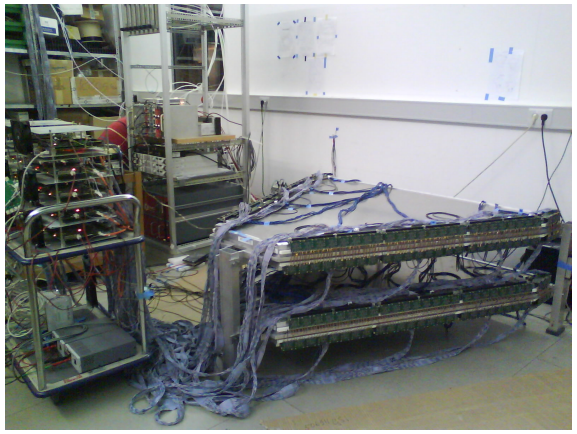

(b)

Figure 1: a) Internal scheme of the detector. RPC shielded cells distribution in rows, columns and layers is shown. b) Two HADES RPC sectors taking cosmic ray data in coincidence during the commissioning of the detectors.

The HADES RPC wall was designed to optimize the performance of the spectrometer in high multiplicity environments. A total area of $7.5 \mathrm{~m}^{2}$, covering the six sectors $\left(1.25 \mathrm{~m}^{2}\right.$ each $)$ is filled with 1116 individually shielded cells of 4-gap timing RPCs. Each sector is distributed in two layers with three columns and 31 cells (rows) within each column [2] as it is shown in Fig.1(a). Each cell has two readout channels, left and right, which provide both timing and charge information. The time and X-position observables are obtained as $T_{r p c}=\left(t_{\text {left }}+t_{\text {right }}\right) / 2, \quad X_{r p c}=\left(t_{\text {left }}-t_{\text {right }}\right)$. $v_{\text {prop }} / 2$, where $t_{\text {left } / \text { right }}$ is the time measured by the left or right channel respectively and $v_{\text {prop }}$ is the signal propagation velocity in the cell. The charge is measured through a charge to width (Q2W) method separately in the left and in the right side. During the cosmic test, pairs of two sectors were stacked horizontally as it is shown in Fig.1(b) at a distance of $350 \mathrm{~mm}$. Detectors were operated indoors under a couple of light roofs offering less than one radiation length width to the incoming showers. The reported average time resolution for individual cells was 77 ps sigma 
and the $\mathrm{x}$-axis reconstructed position resolution was below $12 \mathrm{~mm}$ sigma over all cells [5]. The resolution along the $y$-axis is given by geometry, ranging from $22 / \sqrt{12} \mathrm{~mm}$ sigma for the smallest cells to $50 / \sqrt{12} \mathrm{~mm}$ sigma for the biggest ones.

Calibration and synchronization tasks were done using low multiplicity events in order to avoid contamination by multiple hits in the same cell. Position and charge observables were calibrated by setting offsets in the measured values. Timing synchronization between cells was achieved using a recursive algorithm applied on selected track candidates fulfilling the following conditions:

1. Events should have four hits in total, two of them in the upper sector and two of them in the lower one.

2. Difference of position coordinates of hits in two overlapped cells should be within $5 \sigma$ and charges should be over a safe lower threshold value to completely reject crosstalk hits.

The trigger was done asking for a coincidence between upper and lower sectors. Each recorded event has its own time reference. Then, no absolute time offset could be set and only relative times between hits are available. Under the hypothesis that all selected particles travel at the speed of light, time difference between hits should fulfil

$$
\Delta T=\left(T_{i}-\tau_{i}\right)-\left(T_{j}-\tau_{j}\right)-d_{i j} / c=0,
$$

where $d_{i j}$ is the distance between hits of cell $i$ and $j, c$ is the speed of light and $\tau_{i, j}$ is the time offset of the respective cell. $\Delta T$ has been minimized iteratively and convergence was reached after 5 iterations over all cells. Final mean track time resolution was found to be $\sim 176$ ps sigma, it is shown in Fig.2. In the analysis, $\Delta T$ between hits was asked to be always within $5 \sigma$ to define a track candidate. Fig. 3 shows an example of the time profile of a high multiplicity event after calibration.

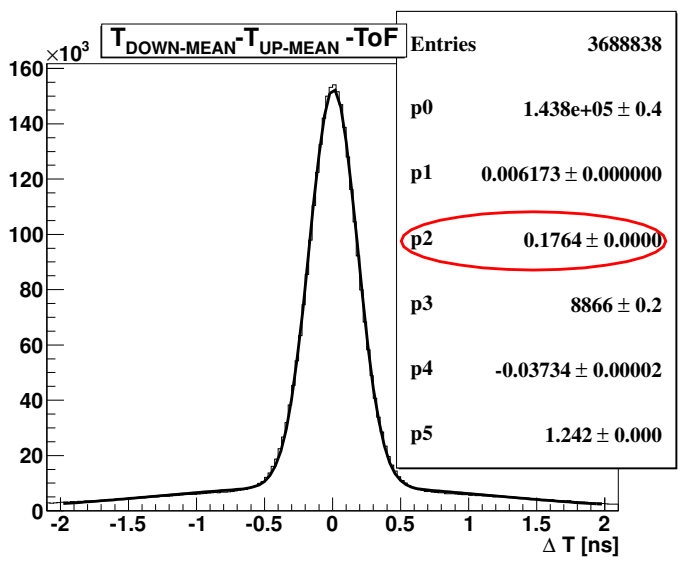

(a)

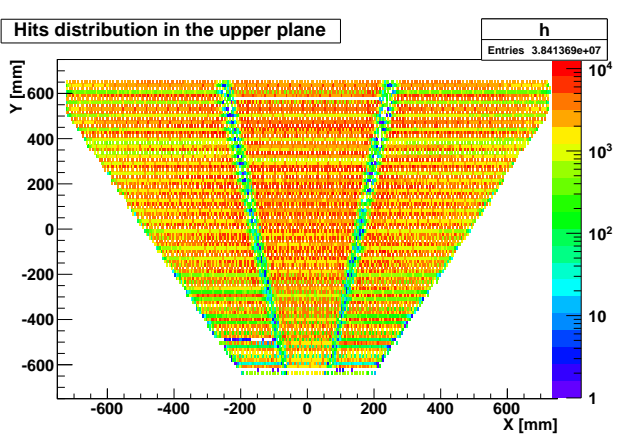

(b)

Figure 2: a) Time synchronization, $\Delta T$, between upper and lower sectors. Two gaussians have been fitted to interpret both signal and background. b) Hit distribution at the upper sector after the position calibration; the three-column structure and different cell geometry is apparent. 


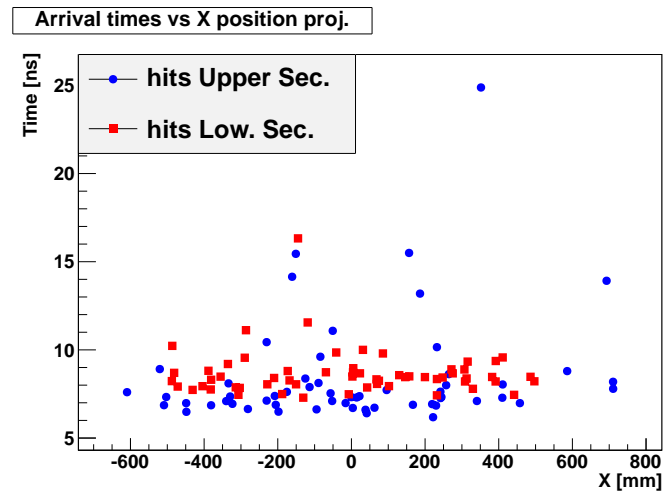

(a)

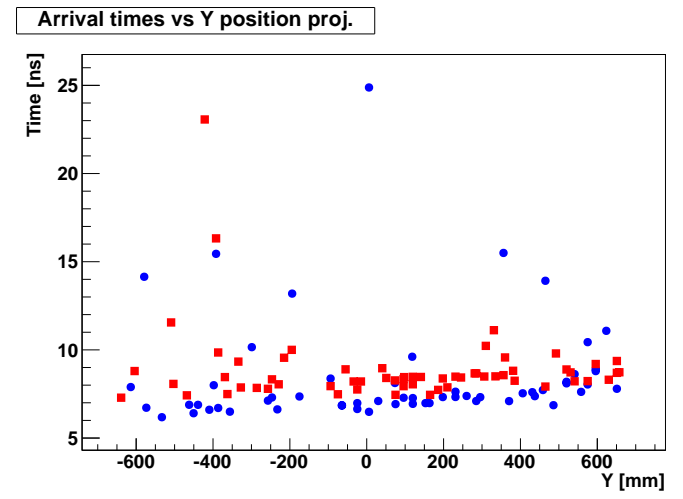

(b)

Figure 3: Example of an arrival profile of a high multiplicity event after calibration; a) Time vs Xcoordinate, b) Time vs Y-coordinate. The incidence angle $\left(\sim 30^{\circ}\right)$ and the characteristic time distribution of a CR shower is apparent. Blue and red markers refers to the top and bottom sectors respectively; $\sim 1 \mathrm{~ns}$ delay due to $350 \mathrm{~mm}$ separation between them is observable.

\section{Results}

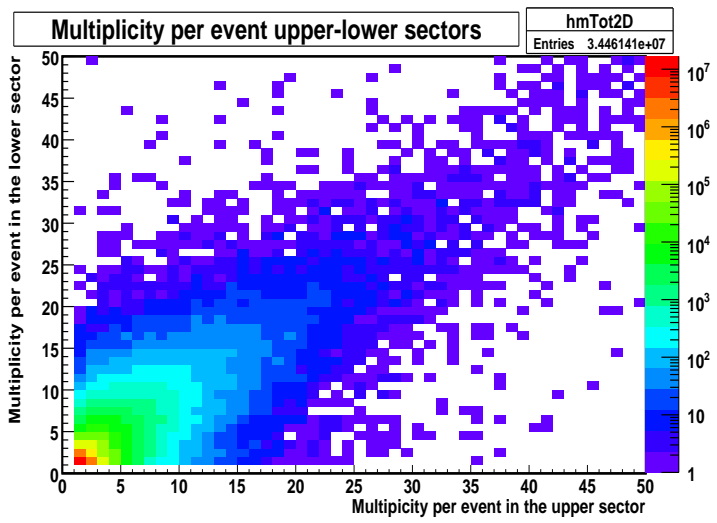

(a)

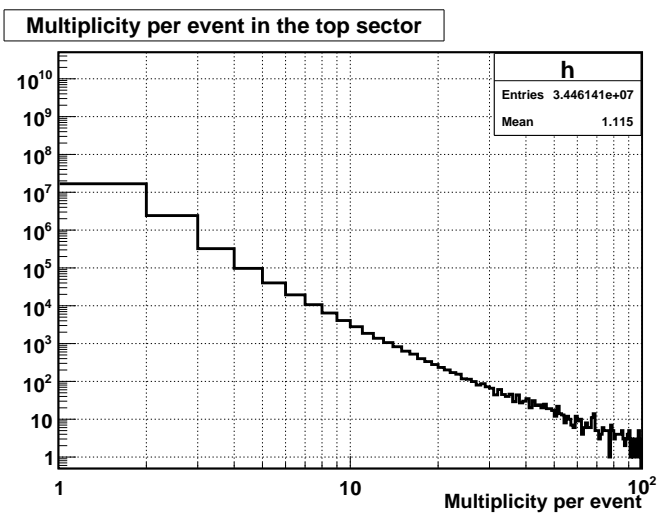

(b)

Figure 4: a) Scatter plot of multiplicities in the upper (x-axis) vs lower (y-axis) planes. b) Measured multiplicity in the upper sector over a continuous five day period.

We present in this section some results showing the capabilities that a high granularity and a high time resolution detector offers to analyse the low scale structure of showers induced by primary cosmic rays. No correction related with the geometry, efficiency, dead time, occupancy or acceptance have been performed.

The multiplicity of the events recorded during the five days period is shown in Fig.4. Fig.4(a) is a scatter plot of the multiplicities recorded at the upper and lower sectors. Fig.4(b) shows the multiplicity per event measured at the upper sector.

Fig.5(a) shows the number of events as a function of both, the track multiplicity and the time width of the front, defined as the time difference between the first and the last arriving tracked 


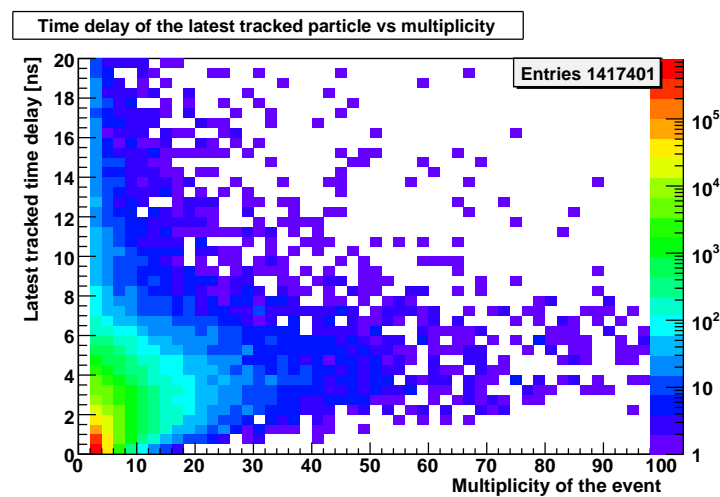

(a)

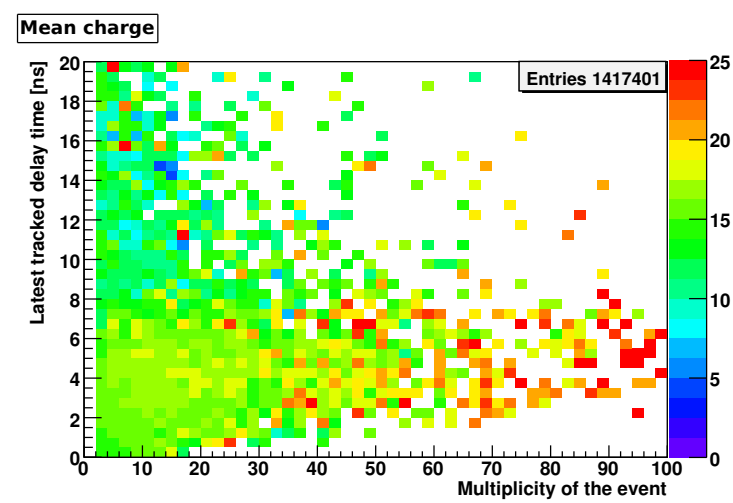

(b)

Figure 5: a) Scatter plot of measured multiplicity vs the latest tracked particle arrival time. Both, multiplicity and the time spread of the events provide information about the energy of the primary CR and the distance to the shower core. b) Same plot representing in the z-axis the mean measured charge in arbitrary units.

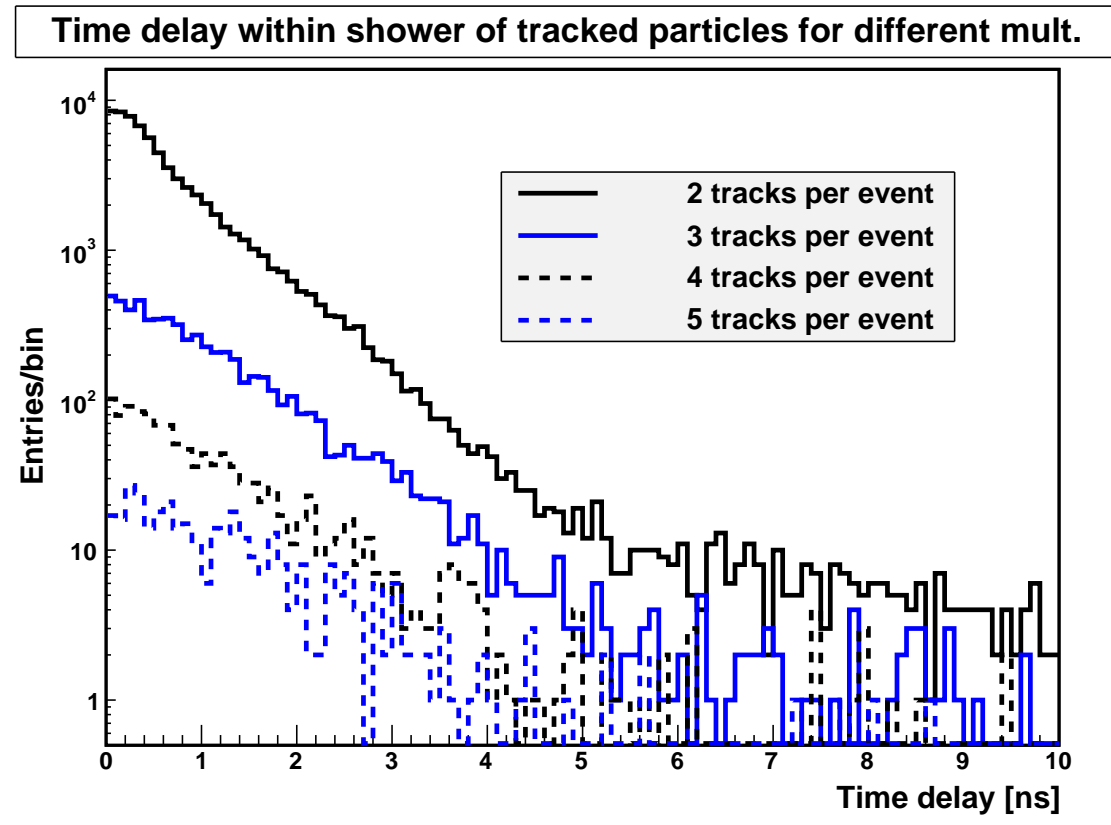

Figure 6: Time delay within shower front to the first reconstructed track. Neither geometry, efficiency, dead time nor acceptance corrections are included. 
particle without any arriving angle correction. Roughly, bigger multiplicities correspond to higher energy primaries and bigger time widths correspond to higher distances between the detector and the core of the shower [8]. Then, low energy showers tend to occupy the bottom-left corner of the figure whereas very much less frequent high energy shower tend to occupy the upper-right corner.

Fig.5(b) shows in the z-axis the mean charge per event in arbitrary units. As electrons are in general more ionizing particles than muons, the figure shows the well known effect that the electromagnetic component dominates near the core of high energy showers (bottom-right side) whereas the muonic component dominates far away from the core (upper-left side). Also the electromagnetic component near the core weakens as far as the showers energy decreases. Some background of electrons or positrons produced either at the supports of the detector or the walls may be present.

Fig.6 shows the time delay of the particles respect the first arriving track for low multiplicity events. In all the cases, the distributions are dominated by time delays that follow exponentiallike distributions. The corresponding scale parameter (widht) increases with the multiplicity of the shower, ranging between $\sim 1$ ns ( 2 track events) and $\sim 4$ ns ( 5 track events). According to the effects observed in Fig.5, this result seem to complement at low energies, the well know behaviour that the time widths of the extended air showers tend to increase with the distance to the core [9]. The background of events visible with time delay bigger than $\sim 6$ ns may correspond to less frequent very high energy showers arriving very far away from the detector.

\section{Conclusions}

Relative small detectors based on several planes of timing RPCs with both high granularity and very good space-time resolution may provide access to a wide range of cosmic ray observables with at an affordable cost: particle density, arrival time structure, particles and shower arrival directions, etc. These kind of detectors may be very useful for cosmic ray studies at a wide range of energies, either working stand alone or as part of hybrid cosmic ray observatories.

\section{References}

[1] P. Salabura, et al., Nucl. Phys. A 749 (2005) 150.

[2] D. Belver et al., Nucl. Instr. and Meth. A 602 (2009) 687.

[3] Ch. Finck, et al., Nucl. Instr. and Meth. A 508 (2003) 63.

[4] H. Alvarez Pol et al, Nucl. Instr. and Meth. A 535(2004) 277.

[5] A. Blanco, et al., Nucl. Instr. and Meth. A 661 (2012) S114.

[6] P. Assis, et al., $R \& D$ for an autonomous RPC station, ICRC2011.

[7] D. Belver, et al., Nucl. Instr. and Meth. A 661 (2012) S163.

[8] W. D. Apel et al., Astropart. Phys. 29 (2008) 317-330.

[9] G. Agnetta et al., Astropart. Phys. 6 (1997) 301-312. 